\title{
Spotlight on measles 2010: A cluster of measles in a hospital setting in Slovenia, March 2010
}

M Grgic-Vitek (marta.vitek@ivz-rs.is) ${ }^{1}$, T Frelih ${ }^{1}$, V Ucakar $^{1}$, K Prosenc ${ }^{2}$, J Tomažič3, M Petrovec ${ }^{4}$, A Kraigher ${ }^{1}$

1. Communicable Diseases Centre, National Institute of Public Health of Slovenia, Ljubljana, Slovenia

2. Laboratory for Virology, National Institute of Public Health of Slovenia, Ljubljana, Slovenia

3. Clinic of Infectious Diseases, University Medical Centre Ljubljana, Ljubljana, Slovenia

4. Institute of Microbiology and Immunology, Faculty of Medicine, University of Ljubljana, Ljubljana, Slovenia

After ten years of being measles free, Slovenia experienced a cluster with secondary transmission in a hospital setting in March 2010. The index case, a resident of Ireland, was hospitalised on the day after his arrival to Slovenia and diagnosed with measles two days later. After his discharge, two cases of measles were notified, a hospital staff member and a visitor to the clinic, suggesting transmission in a hospital setting.

\section{Background}

Measles is a highly infectious disease which can be successfully prevented only by vaccination. Notification of measles cases has been mandatory in Slovenia since 1948. According to the Infectious Diseases Act, a case of measles (even a suspected case) has to be reported within three to six hours to the regional Institute of Public Health, responsible for public health interventions and from there immediately to the National Institute of Public Health (NIPH) where data are collected and analysed. In 2005, the European Union case definition [1] for measles was widely publicised and general practitioners and paediatricians were actively encouraged to confirm every possible case of measles (rash fever) with appropriate laboratory diagnosis.

In Slovenia, mandatory vaccination against measles was introduced in 1968 for 12 months old children. In the first years the vaccination coverage was quite low, but already in 1972 (birth cohort 1971) it reached 60\%. In 1979 the coverage reached $80 \%$ and increased further in the following years. The second dose of measles vaccine was introduced in 1978 for children entering school at the age of seven years (birth cohort 1971), and was replaced by a combined vaccine against measles and mumps in 1979. The coverage for the second dose at seven years of age reached $90 \%$ already in the first year, and has been higher than 95\% since 1983 (data from annual reports of NIPH) [2]. In 1990, the combined measles-mumps vaccine was replaced by a trivalent vaccine against measles, mumps and rubella (MMR); since then children have been immunised with this vaccine at 12 to 18 months (first dose) and at six years of age (second dose).

After the introduction of measles vaccination the occurrence of measles was substantially reduced compared with the highest reported incidence rate of 407 per 100,000 in 1967, and followed a declining trend (Figure 1). The size of epidemics decreased and inter-epidemic periods lengthened. The last case (indigenous) was reported in 1999. The last reported epidemic started in 1994 and peaked in 1995 when 405 cases $(20.4 / 100,000)$ were reported, mostly from two regions of Slovenia.

Before the introduction of measles vaccination in Slovenia, measles was a disease of pre-school children. After that, the age distribution of morbidity shifted to older age groups. The average age of reported cases increased gradually from 5.4 years before the vaccination started $(1965-1968)$ to 11.4 years in the 1990 s (1989-1998) (unpublished data). However, since 1984, an increased proportion of cases has also been observed among infants under the age of 12 months who are not targeted by MMR vaccination (although only seven, nine and 13 cases were reported in 1996, 1997 and 1998, respectively) (Figure 2).

With regard to susceptibility profiles obtained from serosurveys conducted in Slovenia in 1998 and 2000, the population born before 1960 could be considered immune against measles (the proportion susceptible was $1.5 \%$ in those older than 40 years) [3]. Most people borne after 1971 received two doses of measles vaccine. Thus, the cohorts born between 1960 and 1971 would be most at risk of getting measles if the infection was imported to the country.

\section{Cluster description}

On 11 March the NIPH was notified of a suspected case of measles (Patient 1) in a 19 year old resident of Ireland, who was hospitalised in the Clinic of Infectious Diseases at the University Medical Centre Ljubljana 
(CID). On the morning of the same day he was first examined in an emergency outpatient clinic where he presented with an atypical rash (a few abdominal papulae). The patient informed the staff that his brother had been diagnosed with measles a week before and was hospitalised while travelling through Rome, Italy.

\section{FIGURE 1}

Reported measles incidence rates, Slovenia, 1960-2010

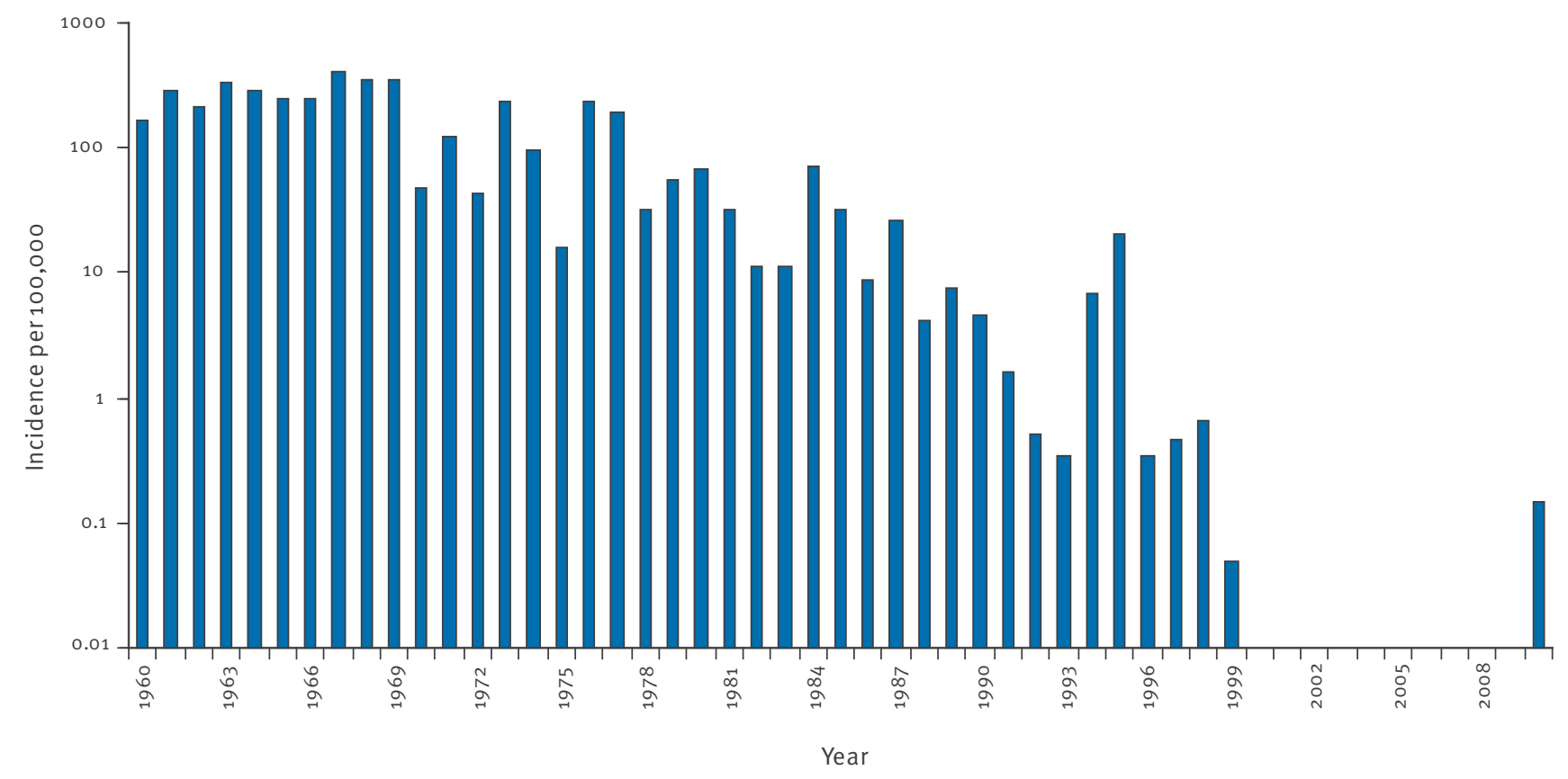

Data as of end April 2010.

Source: National Institute of Public Health of Slovenia.

\section{FIGURE 2}

Age-specific proportions of reported measles cases, Slovenia, 1965-1998

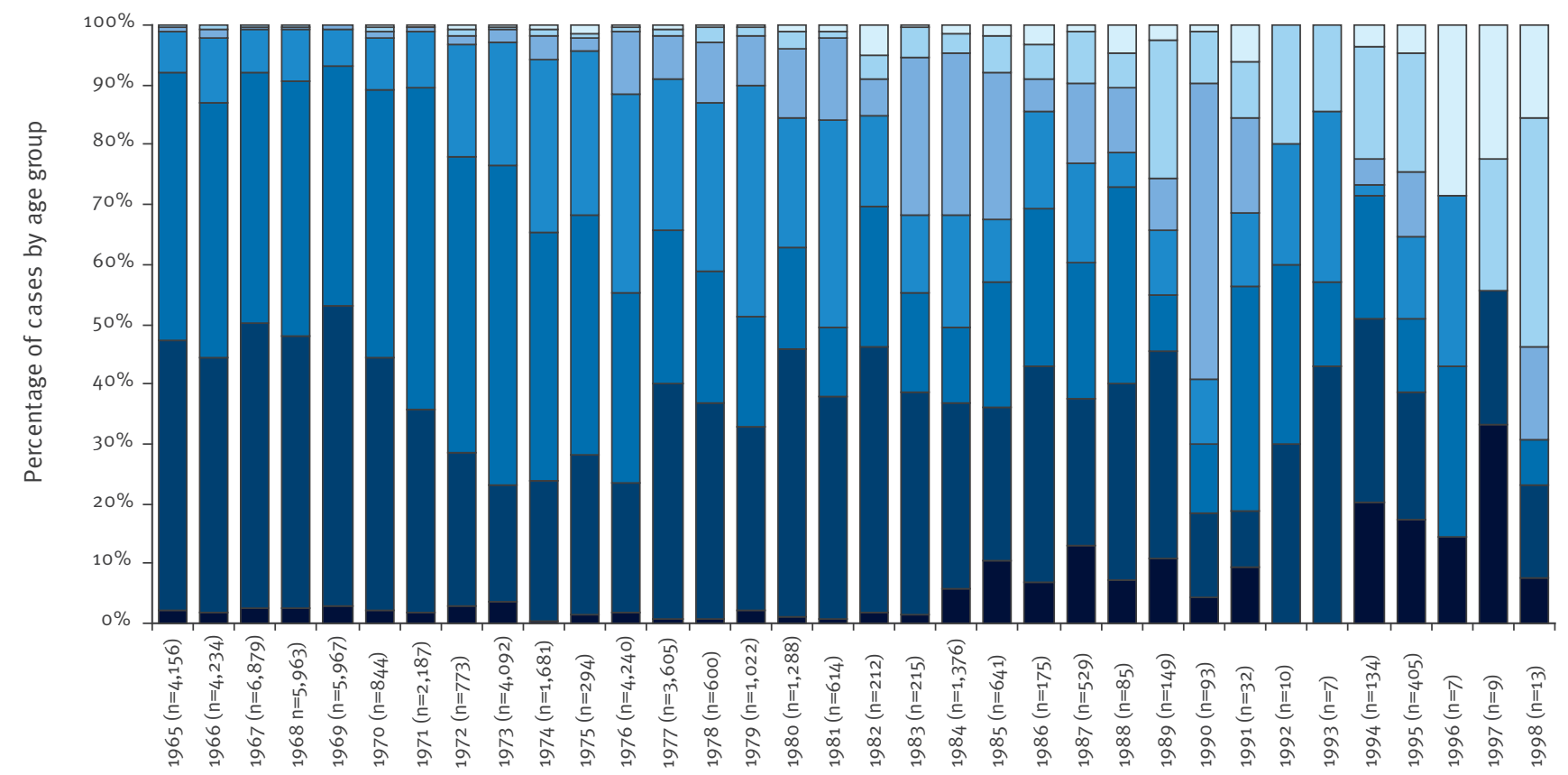

Year and number of cases

Age (years)

$$
\square 3_{30} \quad \square_{20-29} \square_{15-19} \square_{10-14} \square_{5-9} \square_{1-4} \square_{<1}
$$


The brother did not accompany the family to Slovenia. Patient 1 was therefore transferred to CID in the afternoon of 11 March, where he was isolated with fever, a few abdominal papulae, conjunctivitis, and widespread Koplik spots. A blood sample and throat swab (from Koplik spots) were taken on the same day. The patient's serum was tested for measles-specific IgM and IgG by ELISA (Siemens Enzygnost) and was negative for both. In the swab MV was confirmed by PCR of the nucleoprotein gene, and material from the swab was sent for MV isolation and genotyping to the WHO Regional Reference Laboratory for MMR at the Robert Koch Institute, Berlin. The detected MV belonged to genotype $\mathrm{D}_{4}$ and was most similar to MV detected in the UK in 2009. The rash became typical for measles on 12 March and measles-specific IgM resulted positive in another blood sample on 13 March, while IgG was still negative. In the following days the patient's condition worsened and he developed pneumonia. He was discharged from hospital on 19 March fully recovered. In the last specimens taken on that day the IgM titre became lower and specific IgG antibodies appeared (Table).

The patient came to Slovenia with his family in the evening of the day before he was admitted to the hospital. He had no contact with local people as he and his family were sleeping in a caravan. He did not know if he had been vaccinated against measles. According to his statement he was a member of the Irish Traveller community [4] and originating from Limerick, Ireland where an outbreak of measles was ongoing in early 2010.

On 24 March the NIPH was notified of another suspected case of measles (Patient 2) in a healthcare worker who had been in contact with the index case at his admission. The patient had fever, sore throat, muscle aches, vomiting, photophobia, but no typical rash (only a few papulae in the face). At first she was classified as a probable case of measles. She reported to have been vaccinated at least once (as she was born after 1971 she was supposed to have received two doses). Serum specimens taken on 23 and 25 March were tested with ELISA. Both were negative for IgM and positive for $\mathrm{IgG}$ antibodies $(400 \mathrm{IU} / \mathrm{mL}$ ). A throat swab taken on 24 March tested negative for measles with PCR. An archived serum sample taken from this patient six months earlier showed the same titre of measlesspecific IgG $(400 \mathrm{IU} / \mathrm{mL})$ as in the current sera.

Patient 2 was ruled out as a case of measles and therefore was not part of this cluster. Serological evidence (IgG) indicated that the patient was fully protected against measles after being vaccinated as a child, probably with two doses, and her symptoms and signs must have been due to a different viral infection.

Another suspected case (Patient 3) was notified on 1 April in a healthcare worker involved in the care of the index case. According to her self-reported vaccination status she was vaccinated once and was thus allowed to care for Patient 1. When in contact with patients she was always wearing a mask. She was tested for immunity to measles on 16 March (together with other staff members exposed to the index case at his admission) and was found IgG-negative. Nevertheless, she was not excluded from work. She was not vaccinated against measles at that time because she had mild conjunctivitis and herpes labialis (already on 15 March).

On 23 March Patient 3 reported fever, cough and coryza. She noticed a few papulae on her neck and forehead on

\section{TABLE}

Patients notified to the National Institute of Public Health of Slovenia as suspected measles cases, Slovenia, March 2010 $(\mathrm{n}=4)$

\begin{tabular}{|c|c|c|c|c|}
\hline Patient & Status/case classification & Sex, age & Onset of illness & Laboratory results (date of sample taken) \\
\hline 1 & Index case, confirmed & Male, 19 & 11 March & $\begin{array}{l}\text { IgG neg, IgM neg (11 March)IgG neg, IgM pos (13 March) } \\
\text { IgG pos, IgM pos (19 March) } \\
\text { PCR pos (11 March) } \\
\text { Genotype D4 }\end{array}$ \\
\hline 3 & Secondary case, confirmed & Female, 39 & 23 March & $\begin{array}{l}\text { IgG neg (16 March) } \\
\text { IgG pos, IgM borderline ( } 27 \text { March) } \\
\text { PCR neg ( } 8 \text { April) }\end{array}$ \\
\hline 4 & Secondary case, confirmed & Male, 54 & 23 March & $\begin{array}{l}\text { IgM pos, IgG pos (1 April) } \\
\text { PCR pos (1 April) } \\
\text { Genotype D4 }\end{array}$ \\
\hline
\end{tabular}


25 and 26 March and some abdominal papulae on 27 March. A sample taken on 27 March resulted positive for measles-specific IgG $(8,800 \mathrm{IU} / \mathrm{mL})$ and borderline for IgM. She stayed at home for a week from 29 March to 2 April. Throat swab and urine specimens taken on 8 April were PCR-negative (Table).

On 2 April, NIPH was notified of a man in his $50 \mathrm{~s}$ (Patient 4) diagnosed with measles at CID on 1 April. He had visited his physician on 23 March with high fever and malaise. As his condition did not improve he returned on 30 March and was referred to CID due to high gamma glutamyltransferase levels, high levels of C-reactive protein and elevated liver transaminase levels, where he presented on 31 March. Measles was suspected on 1 April, when a typical rash appeared. He had noticed the rash on his neck already on 30 March but not payed attention to it. It was assumed from his age that he was not vaccinated against measles and he did not recall having had the disease as a child. The diagnosis was confirmed by serology (positive IgM and $\lg$ ) and by positive PCR of the throat swab taken on 1 April. Genotyping was performed at the RKI and showed $100 \%$ agreement with the sequence from the MV of Patient 1 (Table).

Between 12 and 21 March (after the isolation of Patient 1), this patient had been visiting twice a day a relative who was hospitalised on the same ward as the index case. He did not travel during or shortly before the incubation period and had no known contact with measles cases. He lives with his wife who had measles in childhood; other members of the family were vaccinated against measles according to the vaccination programme.

An alert was issued on 13 March through the Early Warning and Response System (EWRS) following the diagnosis of the index case. On 2 April the NIPH informed paediatricians and general practitioners about the outbreak through regional epidemiologists; information about measles cases was also published at NIPH website. Guidance for healthcare workers was prepared; an algorithm for the management of measles cases was published on the NIPH website (http:// sm146.slohosting.com/Planet/ ?ni=150\&pi=5\&_5_ Filename $=1246 . \mathrm{pdf} \&$ _5_Mediald=1246\&_5_ AutoResize $=$ false $\& \mathrm{pl}=150-5 \cdot 3$.$) .$

\section{Discussion}

We describe a nosocomial cluster in a highly vaccinated population of Slovenia. Different manifestations of measles were observed, depending on the vaccination status of the patients.

Fortunately, measles in the index case was suspected even before the typical clinical picture appeared. Thus, control measures could have been implemented in time. However, despite this, transmission to two individuals occurred in the hospital setting. The index case was placed in a single room with anteroom in droplet isolation. No air condition was in place. All healthcare workers who were exposed to the index case at admission were tested for immunity against measles and offered vaccination if measles-specific IgG test was negative, but they were not excluded from work. Documented evidence of measles vaccination was not available for all healthcare workers.

It is obvious that Patient 3 was infected by the index case. As she reported to be vaccinated once, but tested negative for measles-specific IgG, she should have been considered a vaccine failure case (primary or secondary) Nevertheless, she was not excluded from work despite her susceptibility and exposure history. The observed rapid IgG antibody response could have been due to secondary immune response $[5,6]$. Rising measles-specific IgG in the absence of IgM in vaccinated cases has been described before [7]. Due to clinical presentation (mild measles) and antibody dynamics, Patient 3 was classified as a case of measles due to vaccine failure. According to some authors, most measles cases in a highly vaccinated population represent vaccine failure and are vaccine-modified cases with a lower transmission potential $[8,9]$. Although it is not very clear whether individuals with a mild illness who do not display the full range of clinical signs of measles are capable of transmitting the virus to susceptible persons, early detection of measles cases especially in healthcare workers is important so that appropriate infection control measures can be implemented in time to reduce the risk of nosocomial transmission.

It is not clear how Patient 4 was infected. To our knowledge, he had no direct contact with the index case. It is not very probable that Patient 3 was the source of infection because the illness in both cases was reported to start almost simultaneously. There is a possibility of indirect transmission from the index case.

In case of suspected measles in a hospital setting it is important to identify susceptible staff (without evidence of vaccination with two doses or laboratory evidence of immunity) who should be excluded from contact with suspected cases. Screening of immunity should be considered. Only staff with documented measles immunity should provide care to a suspected measles case.

\section{Conclusion}

This small outbreak clearly demonstrated the importance of implementing all appropriate control measures in healthcare settings. In addition, high measles vaccination coverage and strong surveillance remain critical to prevent future outbreaks.

\section{Acknowledgements}

We would like to thank Annette Mankertz and Sabine Santibanez from the Robert Koch Institute, Berlin for their generous support, confirming promptly the index case and providing us with all information and control material needed. 


\section{References}

1. Commission decision of 19 March 2002 laying down case definitions for reporting communicable diseases to the Community network under Decision No 2119/98/EC of the European Parliament and of the Council (2002/253/EC). Official Journal of the European Communities 2002:L 86/44. Available from: http://eur-lex.europa.eu/LexUriServ/LexUriServ.do?uri= OJ:L:2002:086:0044:0062:EN:PDF

2. Inštitut za varovanje zdravja. [Institute of Public Health]. Analiza izvajanja imunizacijskega programa v Sloveniji. Letna poročila. [Slovenian immunisation programme analysis. Annual reports.] Available from: http:// www.ivz.si/Mp.aspx? $\mathrm{ni}=106 \& \mathrm{pi}=5 \& 5 \mathrm{~s} i \mathrm{~d}=357 \&$ Pagelndex $=0 \& 5$ groupld $=220 \&$ \& newsCategory $=\& \quad 5$ action $=$ ShowNewsFull \&pl=106-5.0

3. Andrews N, Tischer A, Siedler A, Pebody RG, Barbara C, Cotter S, et al. Towards elimination: measles susceptibility in Australia and 17 European countries. Bull World Health Organ. 2008; 86(3):197-204.

4. Gee S, Cotter S, O'Flanagan D, on behalf of the national incident management team. Spotlight on measles 2010 : Measles outbreak in Ireland 2009-2010. Euro Surveill. 2010;15(9). pii: 19500. Available from: http://www. eurosurveillance.org/ViewArticle.aspx?Articleld=19500

5. Ozanne G, d'Halewyn MA. Secondary immune response in a vaccinated population during a large measles epidemic. J Clin Microbiol. 1992;30(7):1778-82.

6. Huiss S, Damien B, Schneider F, Muller CP. Characteristics of asymptomatic secondary immune responses to measles virus in late convalescent donors. Clin Exp Immunol. 1997;109(3):416-20.

7. Artimos de Oliveira S, Jin L, Siqueira MM, Cohen BJ. Atypical measles in a patient twice vaccinated against measles: transmission from an unvaccinated household contact. Vaccine. 2000;19(9-10):1093-6.

8. Mossong J, Muller CP. Modelling measles re-emergence as a result of waning of immunity in vaccinated populations. Vaccine. 2003;21(31):4597-603.

9. Atrasheuskaya AV, Blatun EM, Neverov AA, Kameneva SN, Maksimov NL, Karpov IA, et al. Measles in Minsk, Belarus, 2001-2003: clinical, virological and serological parameters. J Clin Virol. 2005;34(3):179-85. 